\title{
Les éléments du récit personnel dans les échanges en ligne portant sur les sujets d'actualité : raconter pour mieux comprendre, reprendre pour entrer en dialogue
}

\author{
Personal narratives in online public forums: telling stories \\ for better understanding, referring to others for better dialogue
}

\author{
Joanna Górecka \\ Université Adam Mickiewicz, Poznań \\ jgorecka@amu.edu.pl
}

\begin{abstract}
The paper aims to present the potential, for foreign language students, of the exolingual interactions online in public and open forums. For the analysis of students' commentaries, I apply the observation categories that were first elaborated for the analysis of posts published in press sites. I focus on narrative commentaries in which web users organize phenomena and events discussed in public discourse and try to give them their individual interpretation. I consider this kind of interaction on press sites as contributing to the civic debates: I argue that they can be also integrated in observation categories used to describe and analyze exchanges between students discussing social issues in reference to foreign language media information. In the paper I present two kinds of commentaries: those written by native speakers in public forums and those written by FL students in response to public commentaries.
\end{abstract}

Keywords: glottodidactics, written and asynchronous online communication, forum, online learning, narratives, medias

\section{INTRODUCTION}

La capacité à participer aux échanges portant sur des sujets abordés dans les médias fait partie des exigences discursives caractérisant les apprenants de langue de niveau avancé (CECR, 2001). Pourtant, comme nous avons pu le constater lors de notre pratique enseignante, les activités de discussion telles que l'on propose 
souvent pendant les cours de langue constituent un défi pour nos étudiants et il leur est parfois difficile de se positionner face aux sujets abordés dans l'espace public.

Pour créer un contexte propice à l'initiation des apprenants à la participation active et critique aux activités lors desquelles les locuteurs sont invités à coconstruire des connaissances et pour lesquelles ils se document en consultant surtout les sites d'information sur Internet, nous avons choisi de nous intéresser aux possibilités offertes par les tribunes en ligne qui permettent aux lecteurs de réagir face aux articles publiés et d'échanger les opinions. Nous avançons que la lecture des commentaires accompagnant les articles de presse pourrait faciliter la compréhension des événements qui y sont décrits et que plusieurs parmi les billets publiés, notamment sur les sites modérés, présentent une dimension informative (Doury \& Lefébure, 2006; Amossy, 2011 ; Calabrese, 2014) et pourraient donc stimuler la réflexion sur les sujets débattus. En effet, les recherches (Furstenberg 2001; Hanna \& de Nooy 2009; Lewis \& O'Dowd 2016) montrent que les échanges avec les locuteurs natifs peuvent favoriser, chez les apprenants, à la fois le développement de la compétence de communication et l'acquisition de connaissances interculturelles. Dans le présent article, nous présenterons une piste de réflexion didactique entamée lors de notre travail de recherche visant à élaborer des outils conceptuels et descriptifs pour décrire et évaluer les processus d'apprentissage en ligne et aux objectifs d'enseignement/apprentissage académique. Ainsi s'agira-t-il de rendre compte d'un volet des analyses réalisées, portant sur l'opérationnalité des catégories d'observation proposées pour classer les billets publiés dans les forums ouverts et publics (Duret, 2015) et sur le potentiel didactique des analyses explorant cette grille d'observation. Nous admettons qu'elle peut compléter et enrichir les solutions didactiques appliquées pour les interactions académiques (Newman et al., 1995; Garrison et al., 2001 ; Kuster \& Lameul, 2011 ; Kozan \& Cascurslu, 2018). Le questionnement proposé nous permet d'inscrire le présent article dans la thématique proposée pour ce numéro de la revue (Karpińska-Szaj \& Wojciechowska, 2019 ici même) puisque nous nous intéresserons notamment aux billets contenant des éléments de récits personnels.

Notre intention sera ainsi de montrer comment l'analyse des billets, effectuée avec le recours aux catégories de classification proposées pour les échanges publics (Duret, 2015) peut être utilisée dans le cadre didactique. Tout d'abord, nous soulignons la valeur formative de la consultation des forums publics pour ce qui est de la prise en compte des conduites raisonnées des locuteurs, permettant de fournir aux apprenants FLE des outils d'observation pour les démarches argumentatives, visant l'élaboration personnelle du sens. En effet, il est possible d'observer que les scripteurs réagissant dans un forum ouvert et public interviennent souvent pour reproduire, voire réorganiser l'information présentée dans l'article. L'analyse de leurs billets permet ainsi de mettre en relief leurs visées communicatives : celle de se positionner par rapport à l'événement, de comprendre sa signification et ses 
enjeux où bien celle de rebondir sur l'article pour placer un sujet dans un autre cadre, jugé par l'internaute comme plus propice. La prise en compte de la cohérence et de la complétude des récits qui sous-tendent les interventions individuelles permet aux lecteurs non seulement de juger de la légitimité et de la validité des raisonnements présentés mais aussi de mieux identifier les approches que l'on adapte, dans le discours public, face aux sujets débattus.

Nous argumenterons que le recours à la grille d'observation proposée par C. Duret (2015) pour les tribunes en ligne contribue à donner plus de précision aux critères d'évaluation utilisées de manière systématique en FLE pour apprécier le discours des locuteurs participant à des situations de réflexion collégiale, tels que cohérence du discours ou caractère complet de l'argument ${ }^{1}$. Ensuite, nous avançons que la lecture des commentaires publiés par les internautes dans les sites d'information peut être encadrée en tant qu'une pratique permettant aux apprenants de mieux se documenter, de construire des connaissances sur les sujets débattus dans le pays de la langue cible, de se positionner de manière plus réfléchie par rapport aux arguments formulés dans l'espace public. Observer la qualité du récit devient donc un premier pas permettant ensuite d'introduire un travail d'évaluation portant sur l'efficacité communicative des locuteurs et sur la qualité du sens co-construit.

\section{OBSERVATIONS SUR LE RÉCIT DANS LA RÉFLEXION GLOTTODIDACTIQUE}

Nous insisterons dans cette section sur l'organisation narrative des connaissances individuelles et sur l'importance du dialogue dans les processus d'enseignement/apprentissage. Notre intention est de souligner que, pour que l'interaction scolaire puisse encourager les apprentissages, son organisation devrait prévoir des moments de délibération permettant aux apprenants de vérifier la qualité de leurs interprétations, soit suite à des échanges avec les autres, soit grâce à un travail de réflexion personnel.

\subsection{Considérer le récit dans la perspective dévéloppementale}

Nous avançons dans notre raisonnement que le récit forme une expérience discursive fondamentale pour le développement de l'individu: il organise son expérience et lui permet de comprendre le monde (Nonnon, 2000). De même, comme le remarque J. Trzebiński (2002, p. 33), la structure de l'interprétation narra-

\footnotetext{
${ }^{1}$ Ces critères sont également utilisés dans les recherches portant sur les apprentissages en interaction réalisés dans les forums virtuels (Newman et al., 1995 ; Garrison et al., 2001 ; Kuster \& Lameul, 2011 ; Kozan \& Cascurslu, 2018).
} 
tive permet à l'individu de mieux saisir le contexte et d'insister sur ses particularités dans le processus d'interprétation des faits.

La qualité du récit devient ainsi un enjeu essentiel pour l'enseignement/apprentissage institutionnel puisque, comme le démontrent plusieurs recherches (pour une synthèse, voir Klus-Stańska, 2000) elle influe sur la qualité des raisonnements élaborés. En même temps, la forme du récit dépend également de l'âge de l'apprenant et elle reflète ses compétences, permettant de juger de la stabilité de ses acquis (KlusStańska, 2002, 2010; Nonnon, 2000). En se référant aux travaux de W. Labov², E. Nonnon (2000) remarque que l'efficacité narrative réside dans la capacité du lecteur à mettre en relief l'enjeu et l'intérêt de son récit. On s'attend donc à ce que le locuteur soit capable de rendre sensible la pointe de son histoire - capable d'expliquer aussi bien la visée de son intervention que sa place dans l'échange - et qu'il mette bien en relief la dimension d'inédit de son récit, ainsi que la signification qu'il devrait prendre pour celui à qui il est proposé (Nonnon, 2000).

La réflexion sur les formes d'expressions narratives en contexte scolaire devrait conduire à s'interroger sur les tâches didactiques qui peuvent faciliter et bien cerner l'élaboration des récits complets et cohérents. En même temps, la réalisation des postulats concernant la mise en œuvre d'une forme d'enseignement insistant sur le dialogue réflexif et critique et sur l'importance de l'orientation narrative dans les raisonnements construits par les individus reste un défi didactique dans la plupart des systèmes éducatifs, où le savoir est trop souvent présenté dans des exposés monologaux, laissant peu de place à la négociation du sens individualisée. Nous développerons cette question dans la section suivante.

\subsection{Le récit à l'école}

Les analyses de la communication scolaires ont permis aux chercheurs de poser quelques postulats très importants. Tout d'abord, si on admet que la compréhension repose sur l'attitude active du locuteur, les apprenants devraient être encouragés à évoquer leurs idées personnelles. En effet, comme le remarque M. Eurrutia Cavero (2013) : «Le récit ne prend tout son sens que lors de sa découverte par le récepteur, en fonction de son bagage socioculturel, de ses propres expériences et du contexte dans lequel il se trouve » (p. 106). Ces observations sont confirmées par de nombreuses recherches du courant constructiviste dont les auteurs (Nonnon, 2000 ; Klus-Stańska, 2002, 2010 ; Mezirow, 2001) constatent que l'interaction, lors de laquelle on ne propose pas aux apprenants de formuler les explications mais on les leur fournit toutes faites, mènent à l'élaboration de représentations souvent simpli-

\footnotetext{
${ }^{2}$ W. Labov (1978), La transformation du vécu à travers la syntaxe narrative in Le parler ordinaire. Minuit.
} 
fiées et incomplètes puisque leur cohérence et exhaustivité n'ont été ni testées ni évaluées dans un processus commun d'analyse et d'interprétation. Les mêmes auteurs signalent également (Klus-Stańska, 2002 ; Audigier, 2017) que les enseignants n'insistent pas suffisamment sur les pratiques qui pourraient permettre à l'apprenant de procéder à l'évaluation critique des récits structurant la présentation des sujets dans les manuels ou des exposés oraux préparés par l'enseignant. Citons à cette occasion les travaux de F. Audigier (2017) qui signale que, dans les récits scolaires auxquels les apprenants sont exposés lors des activités en classe, « il n’y a pas assez d'explicitation de l'explication, donc de la validation des relations causales et des raisons qui expliquent les situations étudiées : le sens commun suffit ou semble suffire » (p. 103).

Il semble important de souligner que, pour les jeunes apprenants d'aujourd'hui, ce sont des lacunes au niveau de la compétence scripturale, dues à une faible exposition à ces formes de communication qui rendent les tâches écrites particulièrement ardues. Parmi les faiblesses répertoriées dans les copies écrites analysées, J. Warchala et A. Skudrzyk (2010) rapportent notamment que les scripteurs n'introduisent pas suffisamment les éléments du contexte et que les données descriptives, orientées vers la présentation des acteurs et des événements restent très réduits : on trouve par ailleurs très peu de conduites d'explicitation visant à rendre compte de la structure temporelle et logique du récit. Ce sont plutôt les relations entre les phrases et pas entre les faits qui semblent maintenir, dans les représentations du scripteur, la cohérence du récit.

Après avoir insisté sur l'importance de l'implication personnelle des apprenants dans le processus de construction des connaissances et la nécessité d'une pratique régulière, nous souhaitons, désormais, présenter les arguments mettant en valeur le potentiel des activités de discussion en ligne. Du fait de leur nature écrite et asynchrone, elles offrent un contexte propice à des conduites réflexives lors desquelles les apprenants sont invités à intégrer de nouvelles connaissances dans leur système de connaissances et à contrôler la qualité de leur raisonnement.

2.3. L'acquisition des connaissances générales en langue maternelle et en langue étrangère dans les tâches de la discussion

Nous insisterons, dans la présente section, sur deux particularités de l'enseignement en ligne qui déterminent son potentiel. Tout d'abord, les activités en ligne reposent souvent sur la communication écrite et asynchrone et c'est un contexte où certains processus cognitifs liés à l'élaboration des connaissances peuvent être plus facilement explorés. Deuxièmement, l'espace virtuel offre un contexte pour les activités de groupe, où les apprenants prennent en charge l'organisation du travail et sont responsables des résultats de la tâche. 
Dans notre réflexion, nous nous intéressons aux propriétés des tribunes considérées comme des espaces d'échange, où les locuteurs se positionnent face aux problèmes publics débattus dans les médias (Duret, 2015 ; Calabrese, 2014). Nous nous référons ici aux observations de B. Hanna et J. de Nooy (2009) qui soulignent que, pour les apprenants de langues, la lecture et la publication des billets accompagnant des articles de presse publiés en langue cible peuvent faciliter la compréhension de l'actualité et contribuer au développement des connaissances interculturelles. Nous avançons ainsi qu'il ne suffit pas, dans les processus d'évaluation portant sur la qualité du raisonnement individuel des locuteurs engagés dans les discussion, de se pencher uniquement sur la qualité de l'argumentation ${ }^{3}$ : il faut également s'assurer de la qualité des ressources personnelles qui permettent à l'apprenant, engagé dans un débat portant sur les sujets publics, de se faire un avis et de réagir.

C'est donc cet aspect narratif que nous mettons au centre de notre réflexion et nous choisissions de nous intéresser aux tribunes, une situation de communication où ce sont aussi bien la pertinence des idées que leur mise en forme qui décident de la qualité de l'intervention. Nous nous pencherons notamment sur les indices contenus dans les billets qui permettent de saisir la cohérence et la complétude du récit sous-jacent et - en conséquence - de juger sur cette base de la (bonne) compréhension des phénomènes discutés.

\section{JOINDRE LA COMMUNAUTÉ DE LECTEURS POUR MIEUX ORGANI- SER ET POUR PARTAGER SES CONNAISSANCES}

Dans le présent article, nous nous proposons de réfléchir à l'utilité de recourir, dans le travail de sensibilisation aux particularités de l'apprentissage en ligne, aux catégories d'observation élaborées pour analyser les échanges dans les espaces virtuels publics et ouverts que constituent les tribunes en ligne (Doury \& Léfebure, 2006 ; Calabrese, 2014 ; Duret, 2015). Nous nous référons en particulier aux travaux de C. Duret (2015) qui a proposé une typologie des usages des tribunes en ligne (p. 60) contenant, entre autres, une catégorie qui nous intéresse davantage ici, c'est-à-dire le registre de la narration. Nous la présenterons brièvement ci-dessous.

Le registre de la narration est l'un des sept registres ${ }^{4}$ que C. Duret (2015) a répertorié dans sa recherche. La prise en compte de cet ensemble permet d'identifier

\footnotetext{
${ }^{3}$ La réflexion sur la dimension cognitive de l'activité en ligne est très développée dans le champ de l'apprentissage des langues assisté par ordinateur (ALAO) et plusieurs grilles d'observation ont été élaborées pour saisir et évaluer la qualité des apprentissages réalisés (voir entre autres Newman et al., 1995 ; Garrison et al., 2001 ; Kuster \& Lameul, 2011).

${ }^{4} \mathrm{C}$. Duret (2015) propose une grille d'observation permettant de classer les commentaires issus des sept registres suivants : (1) registre de l'émotionnel ; (2) enrichissement de l'article, du ou des sujets
} 
Tab. 1. Registre de la narration, une catégorie d'observation pour les usages des tribunes en ligne (Duret, 2015, pp. 64-66)

Le registre de la narration renferme les commentaires contribuant à une « synthèse de l'hétérogène » (Ricœur, 1983, p. $128^{5}$ in Duret, 2015, p. 64), c'est-à-dire des commentaires permettant aux intervenants d'ordonner des phénomènes et des événements, de les appréhender dans des séries larges qui débordent la simple occurrence, de leur donner un sens en les reliant à d'autres phénomènes et événements. Il est possible de distinguer plusieurs catégories de billets faisant partie de ce registre :

- digression à portée générale : commentaire inspiré par l'article mais portant sur un autre sujet ;

- mise en perspective du sujet de l'article vers un sujet plus général mais en relation directe avec le sujet de l'article ;

- mise en perspective diachronique rétrospective ou prospective ;

- mise en perspective géographique ;

- synthèse ou rapprochement de plusieurs sources d'information; synthèse des idées et/ou des faits contenus dans l'article ; synthèse d'un ou plusieurs commentaires ;

- synthèse ou rapprochement de plusieurs phénomènes, discours, faits et/ou événements contemporains ;

- hypothèse ou analyse émise dans le but de comprendre un événement, un phénomène ou les intentions d'acteurs sociaux décrits dans l'article ou un commentaire ;

- point de vue normatif sur un état de société, une situation et/ou un phénomène ;

- simulation : projection d'une réalité alternative en considération de faits ou d'un point de vue (Duret, 2015, pp. 64-66).

les intentions des lecteurs publiant les billets dans les tribunes en ligne. L'avantage majeur de la typologie de C. Duret est de proposer des outils pour une lecture des commentaires moins centrée sur leur forme et d'insister sur l'intention du lecteur. On envisage la publication comme un effet en relation à l'activité de lire l'article ou un commentaire : l'intervention est donc considérée comme une réaction du lecteur, une manifestation de sa volonté de se positionner face à un débat social et/ou aux conceptualisations et aux arguments présentés. Les conduites que l'on classe comme narratives dans la typologie décrite présentent plusieurs particularités résultant de la spécificité discursive des échanges en ligne et celle du contrat de communication. Il faut ici tout d'abord insister sur la nature discontinue de l'échange : les lecteurs réagissent aussi bien à l'article qu'aux commentaires mais leurs réactions s'expliquent surtout par le besoin de se positionner et de faire entendre sa voix (Doury \& Léfebure, 2006 ; Mourlhon-Dallies, 2007 ; Amossy, 2011 ; Calabrese, 2014). Le sens des paroles n'est que rarement négocié, même si les interventions proposées offrent des raisonnements plus ou moins aboutis.

qu'il traite ou d'un ou des sujets soulevés par des commentateurs lors des discussions ; (3) registre de la narration ; (4) registre de l'interactionnel ; (5) registre critique ; (6) point de vue personnel et (7) autres.

${ }^{5}$ P. Ricœur (1983), Temps et récit, t. 1. Paris : Éditions du Seuil. 
La dimension «narrative» renvoie aux interventions dont le but est de se positionner face aux manières de décrire et d'interpréter les "phénomènes et les événements » (Duret, 2015) présentés et débattus. Pour que le billet puisse être classé dans cette catégorie, les conduites du locuteur doivent prendre une dimension dialogique, lui permettant de s'exposer à l'évaluation de la part des autres.

Il nous semble important d'insister sur les rapprochements qu'il est possible de faire entre les récits construits dans les interventions écrites en ligne et les récits oraux. Nous nous référons ici aux observations de E. Nonnon (2000) qui portent sur la narration orale et qui, selon cette chercheuse, présentent souvent une forme hétéroclite : les séquences narratives (le récit) s'entremêlent avec les séquences argumentatives (le commentaire). Ce constat est crucial pour notre raisonnement puisqu'il permet de justifier l'intégration des catégories d'observation portant sur les conduites de narration dans les activités visant avant tout une initiation des apprenants à l'apprentissage en ligne dans les tâches argumentatives de la discussion. Rappelons ici que nous avançons, en effet, que la qualité de l'argumentation dépend de la qualité du récit qui sous-tend l'interprétation du contexte de la part de chaque individu.

Nous nous intéresserons, dans la section suivante, à la question de l'intégration des catégories d'observation élaborées par C. Duret dans le travail didactique visant deux objectifs : (1) encourager les apprenants à prendre la parole et à réagir aux idées abordées dans les articles et (2) donner aux apprenants des outils d'observation leur permettant de mieux saisir les éléments d'un récit bien construit et de réaliser une lecture critique de billets publiés par les autres internautes. Nous admettons que la prise en compte de ces aspects permet d'évaluer la qualité de chaque intervention individuelle et de l'envisager comme une contribution au raisonnement co-construit.

\section{COMPTE RENDU DE LA RECHERCHE}

Au cours de notre analyse, nous avons pu constater que la prise de position par rapport aux sujets d'actualité pose plusieurs difficultés aux apprenants de langue. En nous basant sur les difficultés observées, nous admettons qu'il est nécessaire de proposer des catégories d'observation qui pourraient mieux préparer les apprenants à l'exploitation des ressources médiatiques dans leur travail de lecture et de compréhension. Conçu comme l'étape d'un travail de sensibilisation plus long, notre projet offre donc une piste didactique pouvant contribuer à une meilleure préparation des apprenants aux situations d'échange sur les sujets d'actualité. Notre réflexion s'appuie sur les thèses suivantes :

- Le recours à la lecture critique des billets publiés dans le forum public et ouvert peut faciliter - aux apprenants FLE consultant les médias - l'analyse et l'interprétation (et donc la compréhension) des sujets présentés. 
- La mise en avant des éléments du récit dans les interventions des internautes peut aider l'apprenant à intégrer dans son raisonnement les nouveaux éléments, puisés dans le discours des autres, et ceci de façon plus régulière et plus complète. Cela est indispensable pour que les contributions successives dans les échanges en ligne puissent faire avancer la réflexion co-construite.

- La mise en relief de la cohérence, de l'exhaustivité et de la véracité de la structure du récit peut servir de base pour une évaluation plus pertinente de la qualité des arguments. En effet, c'est la qualité du récit qui sous-tend les interprétations proposées permet de valider la légitimité du locuteur et de juger de la fiabilité des informations contenues dans de son billet.

Nous avançons donc, dans notre raisonnement, que les insuffisances au niveau de la reconstruction des faits et de leur contextualisation peuvent diminuer l'efficacité des interventions réalisées ensuite dans le contexte argumentatif d'une discussion. Ainsi, l'introduction des critères narratifs, permettant l'évaluation des récits, peut rendre le travail sur l'initiation des apprenants à la construction des connaissances en ligne plus utile.

\subsection{Questions de recherche et déroulement du travail d'observation}

Le projet effectué a un caractère exploratoire et vise à déterminer dans quelle mesure la prise en compte des conduites appartenant au registre narratif (Duret, 2015) dans les scénarios d'observation utilisés pour décrire l'activité des internautes en ligne peut contribuer à une meilleure sensibilisation des apprenants aux particularités de la communication dans les forums. Le questionnement proposé vise ainsi à donner un encadrement plus efficace au travail didactique dans les espaces virtuels. Nous présentons ci-dessous les deux questions de recherche qui ont orienté notre travail d'analyse des billets :

- Question spécifique 1: Comment l'introduction des catégories d'observation centrées sur les conduites narratives peut aider le lecteur à problématiser le sujet abordé dans l'article et l'orienter vers une lecture critique ?

- Question spécifique 2 : Comment la prise en compte des exigences conceptuelles et rédactionnelles quant à la qualité du récit dans les contributions publiées en ligne peut aider les locuteurs à mieux participer aux situations de co-construction de sens?

Dans notre démarche, nous avons exploré le corpus des données collectées lors notre pratique enseignante. Les données viennent de trois contextes et englobent :

- Des billets publiés dans les forums ouverts et publics, mais en même temps modérés, puisque la participation exige une inscription antérieure (site d'information public, www.francetvinfo.fr). 
- Des billets publiés par les apprenants FLE réalisant ensemble les tâches en ligne dans les forums intégrés à la plateforme éducative Moodle. Les échanges se font dans un groupe restreint des locuteurs qui se connaissent et qui suivent ensemble le même cursus universitaire. L'objectif des discussions est d'explorer un sujet d'actualité politique et sociale, l'échange étant censé engager les locuteurs à justifier leurs positions et à évaluer la qualité de leurs arguments en référence aux standards de la pensée critique (Boisvert, 1999).

- Des billets rédigés par les étudiants en réaction aux contributions des internautes publiés dans les forums publics et ouverts. Les apprenants ont été invités à lire et interpréter les billets et à publier leurs commentaires en réaction aux paroles du journaliste et/ou d'un internaute. Il faut néanmoins souligner que les étudiants n'ont pas publié leurs contributions en ligne, l'activité visait avant tout l'initiation à une pratique discursive nouvelle pour ce public. Le but de la tâche a été de réfléchir aux défis liés à la prise de parole dans les échanges publics et à ceux de s'intégrer dans une communauté discursive partageant les buts et les attentes formelles et sociales envers la forme et le rôle de l'interaction.

La recherche vise donc à tester l'opérationnalité de l'approche adaptée par C. Duret (2015) et à saisir le potentiel descriptif et évaluatif de l'outil analytique et interprétatif proposé par cet auteur. La particularité la plus saillante de la typologie retenue réside, à nos yeux, dans la mise d'accent sur le sens personnel, individuel de l'activité de lire et de publier pour l'individu.

\subsection{Discussion des résultats obtenus}

Nous présenterons ci-dessous (4.2.1) les observations réalisées lors de la lecture des billets publiés par les locuteurs natifs. Dans la section suivante (4.2.2) nous proposerons un compte-rendu de l'étude portant sur l'activité des apprenants, invités à interagir en ligne.

4.2.1. Explorer les éléments du récit personnel dans les contributions des internautes : lecture de la tribune comme une stratégie facilitant la compréhension de l'article

Les contributions des lecteurs qui interviennent dans les forums publics visent avant tout deux objectifs : soit elles s'orientent vers une évaluation du discours des journalistes, portant alors sur le choix du sujet et/ou la manière de le présenter (Duret, 2015; Calabrese, 2014), soit elles proposent un commentaire personnel, permettant à l'individu d'apporter des éléments d'éclairage supplémentaires, offrant 
une occasion pour mieux organiser sa manière de considérer le problème débattu et pour mieux se préparer à aborder ce sujet dans les échanges de face à face (Doury \& Léfebure, 2006).

Dans le travail d'analyse que nous avons réalisé, nous avons souhaité identifier ces billets publiés en ligne qui, selon la typologie proposée par C. Duret (2015), s'inscrivent dans le registre narratif. Il s'agit donc des billets dans lesquels la réaction du lecteur-scripteur se fait avec la volonté de construire une image plus complexe et/ou plus cohérente du sujet débattu, de concevoir des liens entre les événements, de saisir et d'interpréter les intentions des locuteurs. Nous présenterons ci-dessous un exemple du billet que nous avons classé comme appartenant au registre narratif (Duret, 2015). Pour cela, nous rapportons la séquence des trois billets publiés dans la tribune en ligne d'un site d'information public :

\section{Article de référence : "A Nice, ils se baignent dans l'eau potable de la ville "} (30.05.2017, www.francetvinfo.fr)

Billet $1^{6}$, auteur : France Punie

C'est bien pour ça qu'il ne faut mettre de l'eau dans le pastis qu'avec modération !

\section{Billet 2, auteur : Rapaporte}

Toujours les mêmes questions :(

Peut-on encore se baigner dans les rivières ou encore les fleuves (mais c'est plus compliqué) sans devoir enfiler une combinaison javellisée ? Elle est loin l'époque de Tom Sawyer ! Peut-on vraiment boire l'eau du robinet, ou encore se fier à ceux qui nous répètent que oui malgré son arrière-goût ou parfois, ce qui est plus inquiétant à mon avis, son manque, tout court!

Ça dépends où ?...

Billet 3, auteur PBS (s'adressant à Rapaporte)

Ils font des compétions dans la seine pour prouver que l'eau est propre et sans danger pour les humains. Mais en revanche pour les certains poissons en baie de seine... ils sont contaminés et non comestibles. Chercher l'erreur.

En nous référant aux pratiques interprétatives employées par C. Duret (2015), nous considérons, dans la séquence de billets rapportée, le billet 2 (celui rédigé par Rapaporte), comme une intervention proposant une « mise en perspective du sujet de l'article vers un sujet plus général, mais en relation directe avec le sujet de l'article » (p. 64).

Dans ce billet, le locuteur réagit au fait divers présenté dans l'article pour aborder un problème plus large, celui de la manière dont les localités organisent et gèrent leurs réserves d'eau. Nous considérons qu'il est possible de considérer cette

\footnotetext{
${ }^{6}$ Nous rapportons les billets dans leur forme originale, tels qu'ils ont été publiés sur le site, ne changeant rien ni dans les billets publiés dans les forums publics (section 4.2.1) ni dans les billets écrits par les apprenants (section 4.2.2).
} 
intervention comme appartenant au registre narratif : le but de l'individu est de mobiliser ses connaissances afin d'aboutir à une interprétation plus complexe des événements survenus et, dans un deuxième temps, de passer éventuellement à l'étape de l'argumentation menant à leur évaluation. Il rebondit donc sur l'article pour nouer avec des questions plus vastes - celle de la gestion de l'eau et de sa pollution, sa réaction assure ainsi « une mise en perspective du sujet de l'article vers un sujet plus général mais en relation directe avec le sujet de l'article » (Duret, 2015, p. 64).

Lors de notre analyse, tout comme C. Duret (2015), nous avons pu identifier plusieurs billets appartenant au registre narratif ${ }^{7}$, nous pouvons donc constater que la tribune offre un espace pour un travail de réflexion supplémentaire, permettant à l'individu de donner une signification personnelle aux événements présentés : il reprend certaines idées de l'article et propose un cadre qu'il trouve bien adapté pour envisager les faits relatés et pour esquisser des relations logiques entre les acteurs et les actions décrites.

La lecture des commentaires peut donc rendre plus lisibles les faits et les situations qui constituent le quotidien d'une société. Dans le cas des apprenants de langue, cette expérience peut faciliter le travail de compréhension et d'interprétation des faits médiatiques, en ouvrant ainsi une nouvelle porte pour accéder aux sens et aux jugements élaborés par la société de la langue/culture cible. Il s'agit donc d'une expérience qui contribue au développement de la compétence de communication : la capacité à identifier les particularités des récits proposés dans le cadre d'un débat public offre au locuteur des outils d'observation plus précis, lui permettant de juger de la légitimité du scripteur et de la fiabilité de son discours. Parmi les compétences de base pour bien exploiter les commentaires narratifs nous citerons les suivantes :

- viser à reconstituer la trame narrative pour saisir le sens et le poids des événements identifiés comme cruciaux par le locuteur, tels qu'ils expliquent la chute dans la narration et le jugement final porté sur l'événement ;

- observer les relations entre les acteurs et en déduire leurs compétences, leurs droits et leurs devoirs, ce qui permet ensuite de mieux interpréter la signification des faits relatés ;

- observer comment se construit, dans le récit, la généralisation d'une conduite individuelle.

La particularité de cette démarche, de mise pour les usagers réguliers des médias, échappe parfois aux apprenants de langue qui, dans leur lecture adaptent

\footnotetext{
${ }^{7}$ Nous n'avons pas procédé, dans le cadre de notre recherche, à l'analyse quantitative des billets recensés : notre recherche a un caractère exploratoire, or une démarche quantitative inviterait à un investissement important aussi bien au niveau du personnel que du temps alloué à la procédure (Strijbos et al., 2006). C. Duret (2015) rapporte cependant que, dans le corpus qu'il a collecté, les billets narratifs représentent $154 \%$ de l'ensemble, deux autres catégories qui leur succèdent ce sont le registre de l'émotionnel (qui totalise 19,6\% de résultats) et celui d'enrichissement (16,2\%).
} 
alors une perspective dite verbocentriste, en se concentrant surtout sur les aspects linguistiques du message et qui, en plus, ne prennent pas forcément une attitude active et critique envers les articles lus. Or, le lecteur doit se positionner par rapport au texte lu s'il souhaite en retenir certaines informations et/ou les éléments du raisonnement qui y sont présentés. L'exploitation des commentaires publiés dans la tribune permet ainsi de proposer une stratégie quelque peu innovatrice pour les apprenants, en les invitant à une lecture basée sur les éléments d'observation partiellement nouveaux, réalisée dans un contexte communicatif qu'ils explorent peu dans leurs pratiques médiatiques quotidiennes.

\subsubsection{Prendre en compte la qualité du récit sous-tendant les raisonnements personnels comme une démarche soutenant l'apprentissage}

Nous passerons maintenant au deuxième volet du travail didactique réalisé dans le cadre de notre recherche exploratoire. Notre intention a été de mettre en relief les éléments du récit dans les contributions des apprenants participant à une tâche de la discussion online dans deux contextes différents :

- la lecture critique les billets publiés dans un site d'information public et ouvert ainsi que la rédaction d'un billet s'inscrivant dans les échanges des internautes (voir exemple 1) ;

- les activités de la discussion réalisées dans la plateforme éducative Moodle (voir exemple 2).

Le billet proposé comme exemple 1 présente une réaction à l'article publié sur le site www.madmoizelle.com. Le lien vers l'article précis n'a pas été indiqué par l'étudiant. L'étudiant se joint ici à la discussion initiée par un internaute qui, en se référant au passé artistique du chanteur Orelsan, conteste la décision du jury qui a récompensé l'artiste lors des Victoires de la Musique 2018. L'étudiant propose le billet suivant (exemple 1).

Je vais essayer de vous expliquer l'intérêt autour d'Orelsan. Tout simplement c'est la voix de la jeune génération. La voix d'Orelsan? Je ne peux pas vous imposer mon opinion sur ce sujet, c'est l'affaire de goût. La provocation ? Dans l'ere de smartphone, Snapchat, Facebook et Instagram il faut vraiment attirer attention des autres pour atteindre son but. Comment faire ça mieux que par la provocation? Ses paroles abjectes ? Toute la nouvelle génération utilise ce genre de parole. Il est direct, il a des opinions disant " impopulaires » parce que la plupart des gens ont peur de le dire même s'il pense comme Orelsan. Il y a quelques ans, il y avait Stromae qui a choqué tout le monde. Il était aussi critique, même si les enfants, leurs parents et grands-parents écoutaient la musique de Stromae. Orelsan ? « Basique, simple », c'est Stromae des jeunes ! 
La qualification du billet comme narratif se fait sur la constatation qu'il s'agit ici d'une intervention dans laquelle le locuteur reprend différentes informations et en fait une synthèse, pour se positionner face à la question.

Cette classification n'est pourtant pas aisée et ne se réalise pas sans équivoque. Nous avons toutefois rejeté son attribution au registre de « l'interactif » du fait que le locuteur ne s'adresse à aucun interlocuteur précis et que la reprise des paroles des autres est superficielle et partiellement infidèle. Du coup, l'analyse du contenu nous empêche également de considérer le billet comme «apportant une information supplémentaire » puisque les arguments fournis montrent bien que le locuteur ne se réfère pas à la polémique qui a animé les interventions antérieures. Le billet n'est pas non plus critique car on ne peut pas le considérer comme une analyse portant sur les contenus partagés par les interlocuteurs précédents, notamment du fait que l'apprenant ne semble pas se référer aux paroles de la chanson « Saint-Valentin » dont parlent les autres - et qui est à l'origine de la controverse sociale - mais plutôt à la chanson récente et récompensé lors de la $33^{\mathrm{e}}$ cérémonie des Victoires de la musique («Basique »). Et, finalement, ce billet n'appartient pas au registre «point de vue personnel » car il s'agit pour le lecteur d'une vérité qui s'applique à un cadre plus général, son observation personnelle étant censée amener les autres à rectifier leurs interprétations premières.

Dans l'exemple choisi, la reconstruction des événements par l'apprenant ne peut être considérée ni comme complète, ni comme exacte, puisque le locuteur ne se réfère pas - contrairement aux autres intervenants - à la polémique autour de l'artiste due « aux paroles abjectes ${ }^{8}$ » d'une de ses premières chansons. Ainsi, nous pouvons constater à quel point la lecture des documents médiatiques reste un défi pour les apprenants de langue. En effet, la reconstruction correcte des événements impliquerait la nécessité d'une recherche documentaire approfondie, prenant en compte plusieurs documents, souvent éparpillés. Dans le cas étudié, l'analyse du billet ne permet pas de confirmer la bonne compréhension du contexte puisque l'étudiant ne se réfère pas de manière explicite aux faits mentionnés par les locuteurs précédents.

Le billet suivant (exemple 2) vient d'une discussion réalisée par les étudiants sur la plateforme Moodle. La discussion a été effectuée l'année où la loi interdisant de fumer dans les lieux publics a été introduite en Pologne et elle porte sur ses conséquences pour les citoyens.

\section{Forum sur Moodle}

\section{Odp. Fumer au travail}

Milena, tu as touché le problème qui m'irrite vraiment. Au cours des vacances d'été j'ai eu l'occasion d'observer et de participer au conflit entre les fumeurs, les non-fumeurs et le patron au travail. Lorsque beaucoup d'employés sortaient toujours pour fumet et passer

\footnotetext{
${ }^{8}$ La chanson controversée dont discutent les internautes s'intitule « Saint-Valentin ».
} 
trop de temps sans travailler, le patron a interdit de fumer. Cela suscité le conflit parce que touts les fumeurs ont dit que dès le début le patron savait qu'ils fumaient et qu'ils avaient demandé ils pouvaient le faire au travail. Ils ont considéré donc ce changement de la décision injuste. Cependant, pour moi, lorsque je ne fume pas, c'étaient irritant que j'avais toujours plus de travail faute de personnes qui sortaient pour fumer. Je savaient que beaucoup de personnes a commencé de fumer pour avoir plusieurs pauses au travail. Selon moi les situations comme ça ne devraient pas exister, c'est pourquoi il faut interdire de fumer au cours du travail.

Cet exemple nous permet de signaler un défi important que pose - lors des échanges entre les apprenants où l'interaction entre les locuteurs est gérée par eux-mêmes l'interprétation critique des interventions successives. Dans le billet ci-dessus, le scripteur rappelle une expérience personnelle pour soutenir le point de vue défendu. Même si le récit proposé est élaboré de façon correcte, en tant qu'enseignante nous trouvons cependant important de souligner que, dans certaines circonstances, il peut manquer de l'efficacité souhaitée parce que très peu de détails sur les éléments cruciaux du contexte ont été fournis et les informations nécessaires pour identifier et décrire les acteurs et les événements ne sont pas suffisamment développées.

La forme asynchrone et écrite de l'interaction permet de reposer, dans un nouveau contexte, une question centrale pour l'enseignement institutionnel, adressée aux groupes : comment aider les apprenants à veiller à la cohérence et à la complétude de leurs interprétations personnelles ? La typologie des usages proposée par C. Duret (2015) permet d'insister sur la qualité de l'activité individuelle et de préciser les attentes envers l'activité de tout scripteur. Elle met en relief l'importance de l'engagement du lecteur, indispensable s'il veut mémoriser et manipuler les données nouvelles. Le récit renvoie alors à une mise en mots des expériences et des observations personnelles, permettant aux usagers du forum de se positionner par rapport à un problème et d'initier une réflexion à son sujet.

La prise en compte des exigences formelles décidant de la qualité du récit dans les contributions publiées peut donc être considérée comme une démarche privilégiant l'autocontrôle et la gestion critique de la réflexion personnelle : du coup, elle peut être qualifiée comme favorisant le développement de l'attitude autonome de l'apprenant. De plus, le travail de métacommunication, visant à expliciter les récits personnels qui sous-tendent le raisonnement des apprenants est indispensable pour qu'on puisse ensuite travailler sur l'argumentation critique, donc sur une approche qui invite les participants à évaluer les arguments dans la perspective rationnelle. Avant de conclure nos observations, nous tenons à remarquer que, contrairement aux corpus des billets venant des tribunes en ligne (3.2.1), on trouve, parmi les billets rédigés par les apprenants, très peu de billets narratifs. Nous lions ce phénomène aux représentations de la tâche de la discussion en ligne chez les 
étudiants, qui la considèrent plutôt comme une situation où on donne son opinion. En conséquence, très rares sont les conduites basées sur la réflexion qui viserait la mobilisation de ses ressources ainsi que les étapes initiales de la problématisation.

\section{EN GUISE DE CONCLUSION}

Les observations réalisées au cours de notre projet didactique nous ont permis de confirmer l'intérêt des recherches sur la communication électronique dans les espaces de communication publics et ouverts pour une meilleure description des échanges en ligne réalisés dans le contexte scolaire. L'analyse des billets respectant les principes de classement proposés par C. Duret (2015) permet d'extraire du site médiatique plusieurs interventions à dimension informative, pouvant servir à la communauté et aux scripteurs. Elle contribue ainsi à mettre en valeur la lecture des tribunes et ouvre devant les apprenants de langue un nouvel espace pour les échanges interculturels. Le travail réalisé permet ainsi de mieux cerner la spécificité de l'apprentissage en ligne où l'un des motifs majeurs, parmi ceux qui poussent les locuteurs à intervenir, n'est pas la volonté de persuader les autres mais celle de réagir à l'article et de se faire une opinion face à une information nouvelle qui apparait dans l'espace public. Enfin, le travail entrepris confirme que plusieurs outils élaborés et testés dans le contexte online peuvent contribuer efficacement au développement de la réflexion propre au contexte académique où l'un des objectifs d'enseignement/apprentissage proposé est d'aider les étudiants à mieux comprendre la société dans laquelle on vit.

Il reste cependant à souligner que le travail réalisé est de caractère exploratoire : il serait intéressant de composer un corpus plus complexe de commentaires, de procéder à leur classification collégiale à partir des catégories proposées par C. Duret (2015) et d'observer la conformité entre les interprétations multiples, celles de l'enseignant et de l'ensemble des élèves. Ce type d'études permettrait en conséquence de confronter plus efficacement plusieurs besoins identifiés lors de notre travail, notamment celui d'élaborer des descriptifs plus détaillés pour saisir les particularités et les différences entre les sept registres identifiés. 


\section{BIBLIOGRAPHIE}

Amossy, R. (2011). La coexistence dans le dissensus. La polémique dans les forums de discussion. Semen, 31, 25-42. Retrieved from https://journals.openedition.org/semen/9051.

Audigier, F. (2017). Dans la vie et à l'école. Des récits pour connaitre, apprendre, comprendre le monde, agir, décider, choisir en situation. In A. Dias-Chiaruttini, \& C. Coher-Azria (Eds.), Théories-didactiques de la lecture et de l'écriture. Fondement d'un champ de recherche en cheminant avec Yves Reuter (pp. 95-109). Villeneuve-d'Ascq : Presses Universitaires du Septentrion.

Bauer, B., deBenedette, L., Furstenberg, G. Levet, S., \& Waryn, S. 2016. Internet-mediated Intercultural Foreign Language Education: The Cultura Project. In J.A. Belz \& S.L. Thorne (Eds.), Internet-mediated Intercultural Foreign Language Education (pp. 31-62). Boston: Heinle \& Heinle.

Boisvert, J. (1999). La formation de la pensée critique. Théorie et pratique. Canada : De Boeck Université (Ed. du Renouveau Pédagogique).

Calabrese, L. (2014). Paroles de lecteurs : un objet de recherche hybride en sciences du langage. Studii de Lingvisticã, 4, 13-27.

Conseil de l'Europe (2001). Cadre Européen Commun de Référence pour les Langues : Apprendre, Enseigner, Évaluer. Strasbourg : Didier / Conseil de l'Europe.

Doury, M., \& Lefébure, P. (2006). "Intérêt Général », « Intérêts Particuliers ». La Construction de l'Ethos dans un Débat public. Questions de Communication, 9. Retrieved from https://ques tionsdecommunication.revues.org/7922.

Duret, C. (2015). Typologie des usages dans les tribunes en ligne du Journal de Montréal : les articles d'information et chroniques d'opinions revus et corrigés par leurs lecteurs. Communication, Lettres et Sciences du Langage, 9 (1), 53-78. Retrieved from https://clsl. recherche.usherbrooke.ca/.

Eurrutia Cavero, M. (2013). La société contemporaine et les nouveaux genres discursifs : le récit médiatique. Thélème. Revista Complutense de Estudios Franceses, 28, 105-124.

Garrison, D.R., Anderson, T., \& Archer, W. (2001). Critical Thinking, Cognitive Presence, and Computer Conferencing in Distance Education. The American Journal of Distance Education, 15 (1), 7-23. Retrieved from http://www.qub.ac.uk/mgt/papers/methods/contpap.html.

Hanna, B.E., \& de Nooy, J. (2009). Learning Language and Culture Via Public Internet Discussion Forums. Palgrave Macmillian.

Klus-Stańska, D. (2002). Konstruowanie wiedzy w szkole. Olsztyn : Wydawnictwo Uniwersytetu Warmińsko-Mazurskiego.

Klus-Stańska, D. (2010). Dydaktyka wobec chaosu pojęć i zdarzeń. Warszawa : Wydawnictwo Akademickie ŻAK.

Kozan, K., \&. Caskurslu, S. (2018). On the $\mathrm{N}^{\text {th }}$ presence for the Community of Inquiry Framework. Computers \& Education, 122, 104-118.

Kuster, Y., \& Lameul, G. (2011). Un cadre d'analyse multidimentionnel de débats en ligne asynchrones. In E. Nissen, F. Poyet, \& T. Soubrié (Eds.), Interagir pour apprendre en ligne (pp. 25-43). Grenoble : PUG.

Lewis, T., \& O'Dowd, R. (2016) Online Intercultural Exchange and Foreign Language Learning. In R. O’Dowd \& T. Lewis (Eds.) Online Intercultural Exchange. Policy, Pedagogy, Practice (pp. 21-66). Routledge: New York and London.

Mezirow, J. (2001). Penser son expérience. Une voie vers l'autotransformation. Lyon : Chronique Sociale. 
Mourlhon-Dallies, F. (2007). Communication électronique et genres du discours. Glottopol. Revue de Sociolinguistique en Ligne, 10, 11-23. Retrieved from http://glottopol.univ-rouen.fr/tele charger/numero_10/gpl10_01mourlhon.pdf.

Newman, D.R., Webb, B., \& Cochrane, C. (1995). A content analysis to measure critical thinking in face-to-face and computer supported group learning. Interpersonal Computing and Technology, 3 (2), 56-77.

Nonnon, E. (2000). Ce que le récit oral peut nous dire sur le récit. Repères, Recherches en Didactique du Français Langue Maternelle, 21, 23-52.

Strijbos, J.-W., Martens, R.L., Prins, F.J., \& Jochems, W.M.G. (2006). Content analysis. What are they talking about? Computers \& Education, 46, 29-48.

Trzebiński, J. (2002). Narracyjne konstruowanie rzeczywistości. In J. Trzebiński (Ed.), Narracja jako sposób rozumienia świata (pp. 17-42). Gdańsk : Gdańskie Wydawnictwo Psychologiczne.

Warchala, J., \& Skudrzyk, A. (2010). Kultura piśmienności młodego pokolenia. Katowice: Oficyna Wydawnicza Wacław Walasek. 